\section{Military Technical College Kobry El-kobbah, Cairo, Egypt}

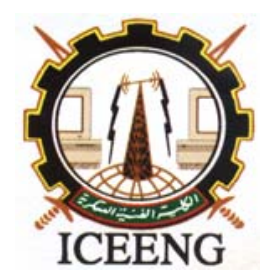

\title{
SIMULATION OF AN ACTIVE RADAR HOMING MISSILE UNDER PHASE-FRONT DISTORTION JAMMING
}

\author{
$5^{\text {th }}$ International Conference \\ on Electrical Engineering \\ ICEENG 2006
}

\begin{abstract}
ALADIN ASSISI*
ABSTRACT

In this paper we introduce a complete modelling and simulation of both target and missile trajectories in one plane with and without the effect of phase front distortion jamming. Although the missile guidance is done in two independent perpendicular planes, the simulation process in the other plane will be identical to that included in this model. Besides, the time variation of different variables in the missile radar guidance system are computed and displayed, to monitor the system performance along the missile trajectory starting from the missile launch up to the impact point. The impact is defined here as the time instant when the rate of variation of the relative speed measured by the missile radio fuse crosses the zero value. This is the same definition adopted by the radio fuse system. The missile-target range at this instant is calculated and displayed as the final miss-distance. Without such a simulation, it is not possible to evaluate an angular deception technique and optimize its parameters; since the instantaneous effect of angle deception will be recovered by the proportional navigation guidance system and the only criterion of jamming effectiveness is the final miss-distance [1]. The simulation program is written as an $\mathrm{m}$-file to be run in MATLAB environment.
\end{abstract}

\section{KEY WORDS}

Target, missile, impact point, radio fuse, miss-distance, jamming effectiveness, guidance, Phase front distortion, induced angular error.

\section{NOMENCLATURE}

$\boldsymbol{a}_{\boldsymbol{r} \boldsymbol{p}}$ repeater gain, target velocity $\boldsymbol{v}_{\boldsymbol{T}}, \boldsymbol{d}$ the distance between the jamming antennas, $\boldsymbol{g}_{\boldsymbol{i}}$ illuminator anenna gain in the target direction, $\boldsymbol{g}_{\boldsymbol{j}}$ the jamming antenna gain, $\boldsymbol{g}_{\boldsymbol{m}}$ missile antenna gain, $\boldsymbol{h}_{\boldsymbol{t}}$ initial heading with respect to the missile direction, $\boldsymbol{l}$ radar wavelength, $\boldsymbol{v}_{\boldsymbol{M}}$ missile velocity, $\boldsymbol{P}_{\boldsymbol{j}}$ the jamming power, and $\boldsymbol{\gamma}$ the voltage ratio between the jamming sources. 


\section{I-INTRODUCTION}

We can evaluate the effectiveness of a deception jamming technique on a tracking radar through mathematical modeling of both the tracking radar and the jammer. The jamming technique can be further evaluated and its parameters optimized through computer simulation. More and above, a combined hardware-software simulation may facilitate fine-tuning of jamming parameters for optimal effectiveness in different operational situations. However, when the tracking radar controls the homing of a missile, a complete theoretical analysis of the problem is no more possible; due to the dynamic variation of all its parameters. The only possible starting point will be to build a computer model for each of the three elements of the problem; namely the target, the missile and the jammer and simulate the interaction of these models to compute the missile trajectory. Each model has to describe, to the most possible accuracy, all the kinetic, kinematics and electromagnetic characteristics of the element it is representing [1].

In a tracking radar jamming problem, we could take the tracking error as an objective function to be maximized. In the missile guidance simulation problem, the tracking error is just one of the dynamically varying parameters. The only possible objective function to be maximized in such a problem is the final miss-distance. Since a complete simulation of the whole missile trajectory has to be done to compute the final miss-distance; such a complete simulation is a single step in the optimization process of jamming parameters. It means that each time a jamming parameter is changed; the whole simulation has to be repeated from missile launch to the impact point.

The author has built this simulation program, called MTJ through a rigorous study and a detailed mathematical analysis of the following:

1. The instantaneous amplitude comparison technique for angle tracking, commonly known as Monopulse Tracking.

2. The Phase Front Distortion technique for angle deception, commonly known as Cross Eye Jamming.

3. The kinematics of proportional navigation technique for missile homing, which is known to be the most efficient homing technique [2]. From references [2 and 3] the ideas for simulating the missile and target kinematics was taken and developed by the author.

4. The kinetics of missile body was not completely modeled in this program. The missile body inertia has been put into consideration by simply inserting a time delay between the generation and the implementation of the guidance commend. This means that the missile normal acceleration waveform has been delayed by $\Delta \mathrm{t}$ with respect to the generated guidance command waveform. The value of this delay is to be selected by the user. One to few seconds are practical values for typical missiles [8].

5. Since the Phase Front Distortion effectiveness is independent of the applied angle tracking technique [5 and 6], the author has built two different simulation models; one based on the complete modeling of the instantaneous amplitude comparison system for angle tracking, which computes the measured viewing angle as such a system does [7], and the other based on the general equation of induced angular error due to phase front distortion jamming, for which the author published a general proof [4]. Simulation runs in different conditions verified the congruence of results of both models.

In the following paragraphs we shall describe briefly the different modules of the MTJ simulation program. 


\section{II- MISSILE-TARGET TRAJECTORY COMPUTATION PROGRAM}

This is the main program, called (MTJ). It computes and plots the target and missile trajectories along the time interval from missile launch to the impact point. The main program calls different modules to compute and plot different tracking and jamming variables at each incremental time step during simulation. The time increment has been selected to be 10 [ms], decreased to $1[\mathrm{~ms}]$ when the missile approaches to 300 meters from the terget, 0.5 [ms] at 50 meters and 50 microseconds at 20 meters to better simulate those critical stages of missiletarget approach.

Since all user interaction is done through this main program, all the parameters of target, missile, tracking and guidance systems and jammer are selected by the user or set as program control parameters.

The program can simulate acttive or semi-active homing as selected by the user. The user can select a typical value for the missile body inertia by selecting the guidance system delay $\Delta t$.

The main program limits the missile normal acceleration to a maximum value of $20 \mathrm{G}$. It provides for a target loss if it goes out of the missile antenna field of view. However, it did not happen in the simulatrion runs that the target was lost.

\section{Target, Missile, Illuminator and Jammer Parameters:-}

The user is asked to input the following target parameters: velocity $\boldsymbol{v}_{T}$, normal acceleration $\boldsymbol{x}_{\boldsymbol{n}} \boldsymbol{T}$, initial heading with respect to the missile direction $\boldsymbol{h}_{\boldsymbol{t}}$, while its radar crossection area $\boldsymbol{\sigma}_{\boldsymbol{T}}$ is a program control parameter.

In semi-active homing, the initial illuminator range at missile launch $\boldsymbol{r}_{\boldsymbol{I T}}$ is a parameter input by the user, while its velocity $\boldsymbol{v}_{\boldsymbol{I}}$ is a program control paremeter.

The following are missile control parameters that can be set by program editing: the missile velocity $\boldsymbol{v}_{\boldsymbol{M}}$, tracking radar wavelength $\boldsymbol{l}$, missile antenna beam width $\boldsymbol{b} \boldsymbol{w}$, antenna squint angle $\boldsymbol{b}_{\boldsymbol{0}}$, missile antenna gain $\boldsymbol{g}_{\boldsymbol{m}}$ and illuminator anenna gain in the target direction $\boldsymbol{g}_{i \boldsymbol{t}}$ The user is asked to select the following jamming parameters:

First he is asked if he wants to simulate with or without jamming. If he selects jamming he is asked if he wants to assume a successful velocity gate stealing. In such a case, the guidance radar does not receive any signal from the target and the jamming-to-signal ratio is infinite. The user is asked in this case to input the value of speed error induced by the jammer in the tracking system $\boldsymbol{d} \boldsymbol{v}_{\boldsymbol{c} j}$. Finally, he is asked to select either a complete simulation of instantaneous amplitude comparison for angle tracking or the direct application of the phase front distortion jamming equation [4]:

$$
\tan \left(\beta_{j}\right)=\left(\frac{\alpha_{j}}{2}\right) \cdot\left(\frac{1-\gamma^{2}}{\gamma^{2}+2 \gamma \cos \varphi_{e f f}+1}\right)
$$

where

$\alpha_{j}$ is the angular separation between the two jamming antennas from the missile's point of view. We shall call it the source resolution angle. It is given by:

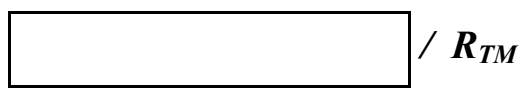


$\lambda$ is the viewing angle of the target center from the missile's point of view $\boldsymbol{h}_{\boldsymbol{t}}$ is the target heading with respect to the negative $\mathrm{x}$ axis (reference direction).

$\boldsymbol{R}_{T M}$ is the Target-Missile range [m]

$\left(\varphi_{\text {eff }}\right)$ is the effective phase difference between the two jamming signals at the missile receiver input, which is given by:

$$
\varphi_{\text {eff }}=\psi+\varphi_{12}=\left(\frac{2 \pi d \sin \left(\lambda+h_{t}\right)}{l}\right)+\varphi_{12}
$$

$\psi$ is the additional phase shift due to path difference between the two waves $\left(\varphi_{12}\right)$ is the intended phase shift between the two coherent sources

$\boldsymbol{l}$ is the wave length [in meters].

An additional set of jamming control parameters are the voltage ratio between the two jamming sources $\boldsymbol{\gamma}$, the distance between the jamming antennas $\boldsymbol{d}$, the jamming power $\boldsymbol{P}_{\boldsymbol{j}}$, the jamming antenna gain $\boldsymbol{g}_{\boldsymbol{j}}$, the polarization match factor $\boldsymbol{k}_{\boldsymbol{p}}$ and the repeater gain $\boldsymbol{a}_{\boldsymbol{r} \boldsymbol{p}}$.

\section{Main Program Description:- Initiallization:-}

A Cartesian set of coordinates with fixed directions and moving center is used. The center is located at the missile. The $\mathrm{x}$ axis is taken in the direction of the missile-target vector at the missile launch. It means that the viewing angle starts at a zero value (See Fig. 1). At $t=0$ target coordinates $\boldsymbol{R}_{\boldsymbol{T M}}$, and $\lambda$ are computed.

From the collision triangle shown in Fig. 3; it is evident that :

$$
\frac{V_{T}}{\sin (L)}=\frac{V_{M}}{\sin (\lambda)}
$$

Thus the initial lead angle $\mathrm{L}$ can also be computed. The missile velocity vector will be inclined to the $\mathrm{x}$ axis by an angle $\theta=\boldsymbol{\lambda}+\boldsymbol{L}$. Both missile and target velocity vectors are resolved into their $\mathrm{x}$ and $\mathrm{y}$ components and the $\mathrm{x}$ and $\mathrm{y}$ components of the relative speed is computed. The closing velocity $\boldsymbol{v}_{\boldsymbol{c}}$ is obtained from the following relation:

$$
\begin{aligned}
& x_{T M} X_{T M}^{*}+y_{T M} \cdot y_{T M}^{*}=-r_{T M} \cdot r_{T M}^{*}=-r_{T M} \cdot \nu_{C} \\
& \text { where } \dot{X}_{T M}^{*} \text { denotes the time derivative of } x_{T M} .
\end{aligned}
$$

\section{Computing target and missile trajectories:-}

The target trajectory is computed directly from its above mentioned parameters. It is independent of the missile trajectory and all the factors affecting it. On the other hand, the missile trajectory depends on target trajectory and jamming effect, as will be shown. The basic idea of trajectory computation is the following:

1. From the target and missile coordinates at a certain time instant $t_{i}$, compute the missile-target range $\boldsymbol{R}_{\boldsymbol{T M}}$ and the real viewing angle $\lambda_{\boldsymbol{r}}$. If there is jamming, the 
measured viewing angle $\lambda_{m}$ is computed by subtracting the jamming induced angular error $\Delta \beta_{j}$

$$
\lambda_{\text {measured }}=\lambda_{\text {real }}-\Delta \beta_{j}
$$

2. This measured viewing angle is the starting point at the next instant $t_{i}+\Delta t$, when the guidance computer generates its next command. In a proportional navigation homing system, the guidance command is proportional to the rate of variation of the measured viewing angle with time. The missile will get a normal acceleration $\mathrm{n}$ proportional to this rate.

$$
a_{n}=N^{\prime} \cdot \dot{\lambda}_{m} \cdot v_{c}
$$

where

$$
\begin{aligned}
& \dot{\lambda}_{m}=\frac{d \lambda_{m}}{d t}=\text { the measured line-of-sight rate of change } \\
& N^{\prime}=\text { effective navigation ratio; } \text { a proportionality constant between } 3 \text { and } 5 .
\end{aligned}
$$

3. The program computes the missile next position when it moves with this new normal acceleration. It computes, also, the new target position at the next time instant. From this data, a new value of target and missile positions, $\boldsymbol{R}_{\boldsymbol{T M}}$ and $\lambda_{r}$ are calculated, to which $\Delta \beta_{j}$ is added. The new value of normal acceleration is computed, and so on, until the missile arrives at its impact point.

4. The simulation ends when the closing velocity $\boldsymbol{v}_{\boldsymbol{c}}$ becomes negative; which means that the missile has arrived at its minimum distance from the target and that their relative range started to increase. At this instant the radio fuse, and consequently, the missile itself, should explode.

5. The simulation program computes and displays $\boldsymbol{R}_{\boldsymbol{T M}}$ at this time instant and call it the miss-distance.

\section{III- CROSS-EYE JAMMING SIMULATION MODULE (CEJ)}

The main program calls this module to compute the results of cross-eye (phase front distortion) jamming each time the target and missile coordinates are computed; i.e. every time interval $\Delta$ t. The calling parameters are: $\boldsymbol{t}, \boldsymbol{R}_{I T}, \boldsymbol{R}_{T M}, \lambda_{\boldsymbol{r}}$ and $\boldsymbol{h}_{\boldsymbol{T}}$. The module computes the following results:

- The jamming induced angular error $\left(\boldsymbol{\beta}_{\boldsymbol{j}}\right)$,

- The angular difference between the two jamming sources seen by the missile $\left(\boldsymbol{a j}_{2}\right)$. In order to get effective jamming the induced angular error must vary with time [1]. Different laws of time variation are available in this module. The default selected law is linear. However, any other law can easily be selected as a control parameter in this module.

- The relative phase difference between the two sources $(\varphi)$ and their effective phase difference in case of semi-active homing $\left(\boldsymbol{\varphi}_{\text {eff }}\right)$, where the path differences due to the target aspect angle are taken into consideration 
- The amplitude ratio of the two jamming sources $(\gamma)$.

- The target-scattered signal power at maximum antenna gain at the missile receiver input $(\boldsymbol{S})$. The computation is done for active or semi-active guidance systems according to the selected case.

- The jamming power at the missile receiver input $(\boldsymbol{J})$. If the repeater jammer arrives at its saturation range, the saturated jamming power is computed and substituted in the equation for $\boldsymbol{J}$.

- A special simulation factor (k1), used for the conversion of the closed loop angle tracking system simulation (when the user selects to simulate the missile angle tracking system). $\mathrm{K} 1$ is a factor multiplied by the resulting error voltage $\boldsymbol{a}_{\boldsymbol{m} \boldsymbol{1}}$ and fed back to the angle tracking system as an iteration step for the angle $\boldsymbol{\beta}$. Calling the AM1M module (or the AMGC module) once more with $\boldsymbol{\beta}$ decremented by the product $\left(\boldsymbol{a}_{\boldsymbol{m} \boldsymbol{1}} \boldsymbol{*} \boldsymbol{k} \boldsymbol{1}\right)$ we get a smaller value of error voltage. The process is repeated until the error is nearly zero. The simulation module (CEJ) increases the value of $\boldsymbol{k} \boldsymbol{1}$ to get faster convergence if the resulting variation of error voltage with respect to the previous iteration is too small. It decreases its value if the new error voltage gets a sign opposite to that of the previous one; which means a zero crossing. The iteration process continues until the resulting error voltage becomes smaller than a given value; which means that the angle tracking system gets its stable equilibrium position. The resulting value of $\boldsymbol{\beta}$ is that of the system equilibrium.

In order to compute the value of $\boldsymbol{\beta}$ at which the tracking system gets its stable equilibrium this module calls another module called AM1M or another module called AMGC.

\section{IV- INST. AMPLITUDE COMPARISON SIMULATION MODULE}

This instantaneous amplitude comparison tracking simulation module, called (AM1M), simulates the performance of the instantaneous amplitude comparison system for angle tracking used in the guidance radar of the active homing missile. It computes the normalized error voltage of the angle tracking system

$$
a_{m}=\left(a_{R}-a_{L}\right) /\left(a_{R}+a_{L}\right)
$$

where $a_{R}$ and $a_{L}$ are the outputs of the Right and Left receiving channels, respectively.

The AM1M module uses all the given parameters of the instantaneous amplitude comparison tracking system, together with all those of the target and jamming signals and the relative viewing angles of both jamming sources and the target body with respect to the antenna boresight, considering the squint angle of both the Right and Left antennas to compute its output error voltage under the effect of phase front distortion jamming. Each time this module is called, it computes each received signal according to its relative amplitude and direction with respect to the direction of maximum gain. The antenna gain function is simulated by a special sync function (g1). The module vectorially sums the input signals at each of the Right and Left receiving antennas, computes the voltage amplitude of each antenna output, and computes the voltage sum and the difference of received signal amplitudes. 
In case of a successful velocity gate stealing, no target signal exists and the receiver input is only the resultant of the two jamming sources. However, if there is no successful velocity gate stealing, the signal scattered from the target body is computed and vectorially summed to the two jamming signals. Naturally, in case of no jamming, the only input will be the target signal. The module exactly simulates the angle tracking system of the missile and computes its output in each of these three cases.

\section{Inst. Amplitude Comparison Tracking Simulation Module ( $A M G C)$}

This is an alternative to the (AM1M) module. Its function is the same, except for the method adopted in the computation of the error voltage. Instead of normalizing the difference signal by the sum signal, it simulates a closed loop automatic gain control system on the sum channel to generate a gain-controlled sum signal. This gain controlled sum is multiplied with the difference signal to give a final result congruent with that of the AM1M module.

When the error voltage resulting from the AGC sum channel is multiplied with the difference channel voltage the result is similar to that of normalizing the difference channel output by the sum voltage; since the AGC output has in its denominator the sum voltage squared (See ref. [5]).

This AGC technique described and analyzed in ref. [5], [9] and [10] has more practical implementation than the normalization technique described in ref. [8] and other literature. However, simulation runs proved that the results of both modules are equivalent.

\section{The Antenna Gain Function G1}

Two different functions for the missile receiving antenna have been simulated. The first (g1) is a sync function given by:

$$
\begin{aligned}
& G=G_{m} \sin x / x \\
& \text { where } \\
& x=(2.783 \beta / \mathrm{bw}) \\
& \text { bw = antenna beam width }
\end{aligned}
$$

The other is an exponential function given by:

$$
\begin{aligned}
& \mathrm{G}=\mathrm{G}_{\mathrm{m}} * \exp \left(-(\beta / \mathrm{k})^{2}\right) / 10 \\
& \text { Where } \\
& \mathrm{k}=.6 * \mathrm{BW}
\end{aligned}
$$

Both antenna models give similar results inside the main lobe. However, the first one is preferred because it better simulates the antenna side lobes, while the second model has no side lobes [11].

\section{V- SIMULATION RESULTS}

Hundreds of simulation runs have been done in different situations, with different values of target acceleration and heading and different jamming parameters. Through those runs, the jamming parameters have been optimized for maximum possible miss-distance. Many Simulation runs showed that the results of complete simulation of the tracking system are the 
same as those of applying the equation (1). We have succeeded to get miss-distances of the order of several kilometers in different cases.

An example run of the simulation program has been done for demonstration in this paper with the following input variables and parameters:

Homing: active, control system delay $[\mathrm{sec}]=1$, target velocity $=250[\mathrm{~m} / \mathrm{s}]$, target acceleration $=-5\left[\mathrm{~m} / \mathrm{s}^{2}\right]$, control system delay $=1[\mathrm{sec}]$, target heading $=10\left[^{\circ}\right], \mathrm{R}_{\mathrm{IT}}=20[\mathrm{~km}]$ Jamming $=\mathrm{Y}$, successful VGPO $=\mathrm{Y}, \mathrm{VGPO}=\mathrm{Y}$, induced speed error $=-100[\mathrm{~m} / \mathrm{s}]$

$\mathrm{R}_{\mathrm{TM}}=20000[\mathrm{~m}]$ at the missile launch

$\mathrm{R}_{\mathrm{TM}}=624.8335[\mathrm{~m}]$ at the impact point

Fig. 4 shows the simulated target and missile trajectories, while Fig. 5 shows some monitored performance variables of the missile guidance system and the jamming parameters during simulation. These variables are

- the real and measured viewing anges $\lambda_{\text {real }}$ and $\lambda_{\text {measured }}\left[^{\circ}\right]$. You can notice that the difference between them is the induced angular error $\boldsymbol{\beta}_{j}=\lambda_{\text {real }}-\lambda_{\text {measured }}\left[^{\circ}\right]$.

- the induced error $\boldsymbol{\beta}_{j}$, computed by the equation (1) or by the tracking system simulation.

- rates of variation of the real and measured viewing anges $d\left(\lambda_{r}\right) / d t$ and $d\left(\lambda_{m}\right) / d t\left[{ }^{\circ} / \mathrm{sec}\right]$

- the effective phase difference between the two jamming sources $\boldsymbol{\varphi}_{\text {eff }}\left[{ }^{\circ}\right]$

- The missile normal acceleration $\boldsymbol{a}_{\boldsymbol{n}}$, computed according to the equation (6) (in blue color) and the implemented one (in red color) delayed by the system delay insrted by the user (1 second in this run).

- The amplitude ratio between the two jamming sources $\boldsymbol{\gamma}=0.964$.

\section{VI- CONCLUSION}

1. A detailed computer model has been designed for the effect of Phase Front Distortion deception technique on a proportional navigation homing missile. The model describes all the details of the tracking radar, the guidance kinematics and the jamming technique.

2. A modular simulation program has been designed, implemented and run which computes and plots different important performance variables of the guidance system under jamming effect; which facilitates the optimization of jamming parameters.

\section{REFERENCES}

1. Aladin H. Assisi, "Simulating And Maximizing The Effect Of Jamming On Proportional Navigation Homing Guidance", $8^{\text {th }}$ International Conference on Aerospace Sciences \& Aviation Technology, May 1999.

2. Zarchan, "Tactical and Strategic Missile Guidance", American Institute of Aeronautics and Astronautics, 1994.

3. G. Biernson, "Optimal Radar Tracking Systems", John Weiley \& Sons, Inc., 1990.

4. Aladin Assisi, "Analysis Of The Angle Tracking Error Induced In A Radar Homing Missile By Phase-Front Distorted Jamming", 9 ${ }^{\text {th }}$ International Conference on Aerospace Sciences \& Aviation Technology, May 2001. 
5. S. A. VAKIN and L. N. Shustov, "Principles of Jamming and Electronic Reconnaissance", Soviet Radio, Translated by the American Intelligence in 1970.

6. Leroy Van Brunt, “Applied ECM, Volume I”, EW Engineering, Inc., 1980.

7. Nader Wagih Abou-Kerdeh, "Performance Evaluation of Angle Deception Missile Jamming System", a PhD. Thesis, MTC, 2001.

8. M. Skolnik, Radar Handbook, McGraw-Hill, New York, 1970.

9. August Golden Jr., "Radar Electronic Wrfare", AIAA Education Series, 1987.

10. M.V. Maksimov, "Electronic Homing Systems", translated by W.F. Barton, Artech House, 1988.

11. Aladin H. Assisi, "Angle Deception of Instantaneous Amplitude-Comparison Radar Tracking System by Phase Front Distortion, Proceedings of the $8^{\text {th }}$ ASAT Conference , Cairo, 1999.

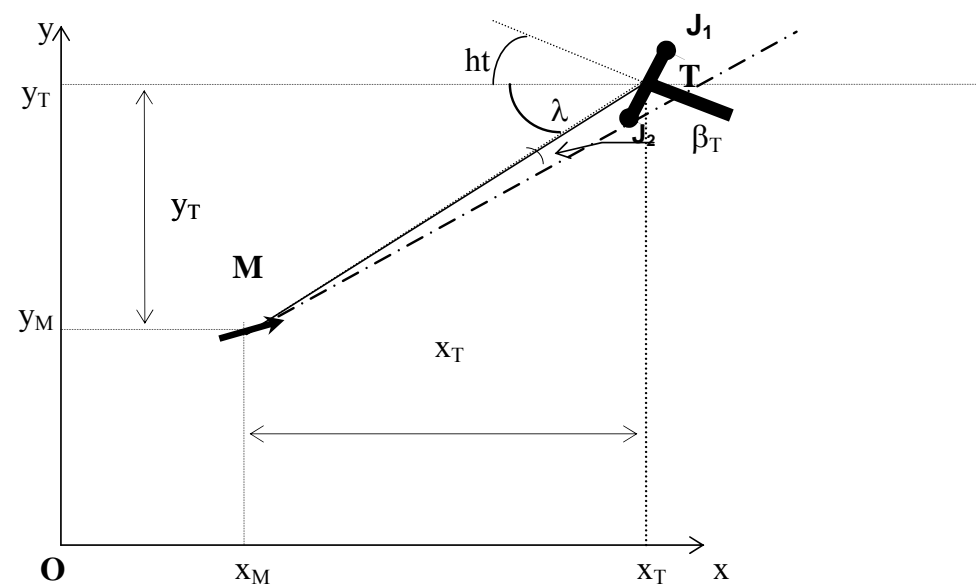

Fig.1 Basic Relations in the Guidance Coordinate System

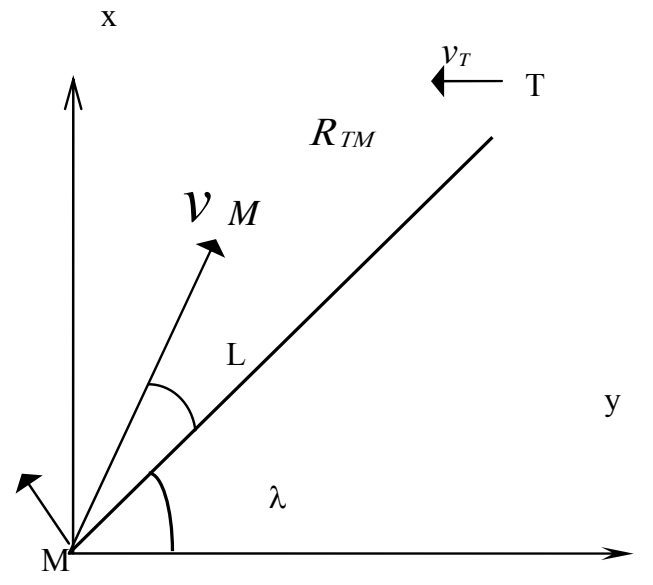

Fig.2. Homing Guidance Kinematic Model

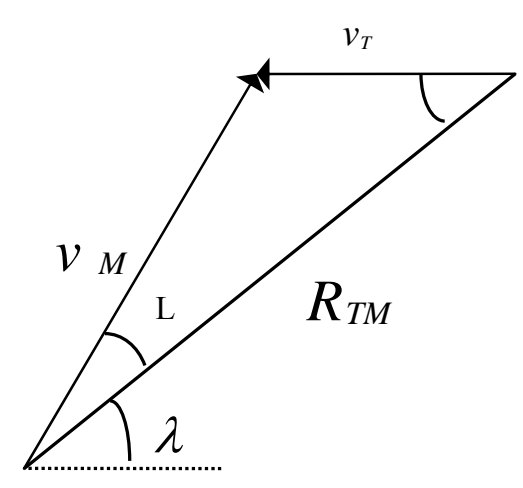

Fig.3. The collision triangle 


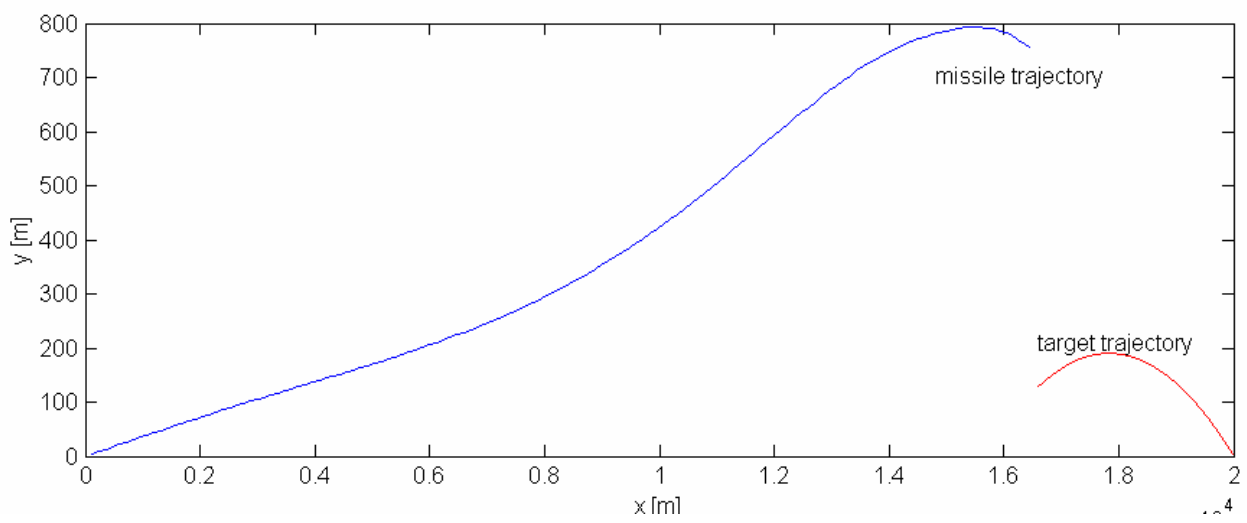

Fig. 4

Target and Missile Trajectories for the Given Input Parameters
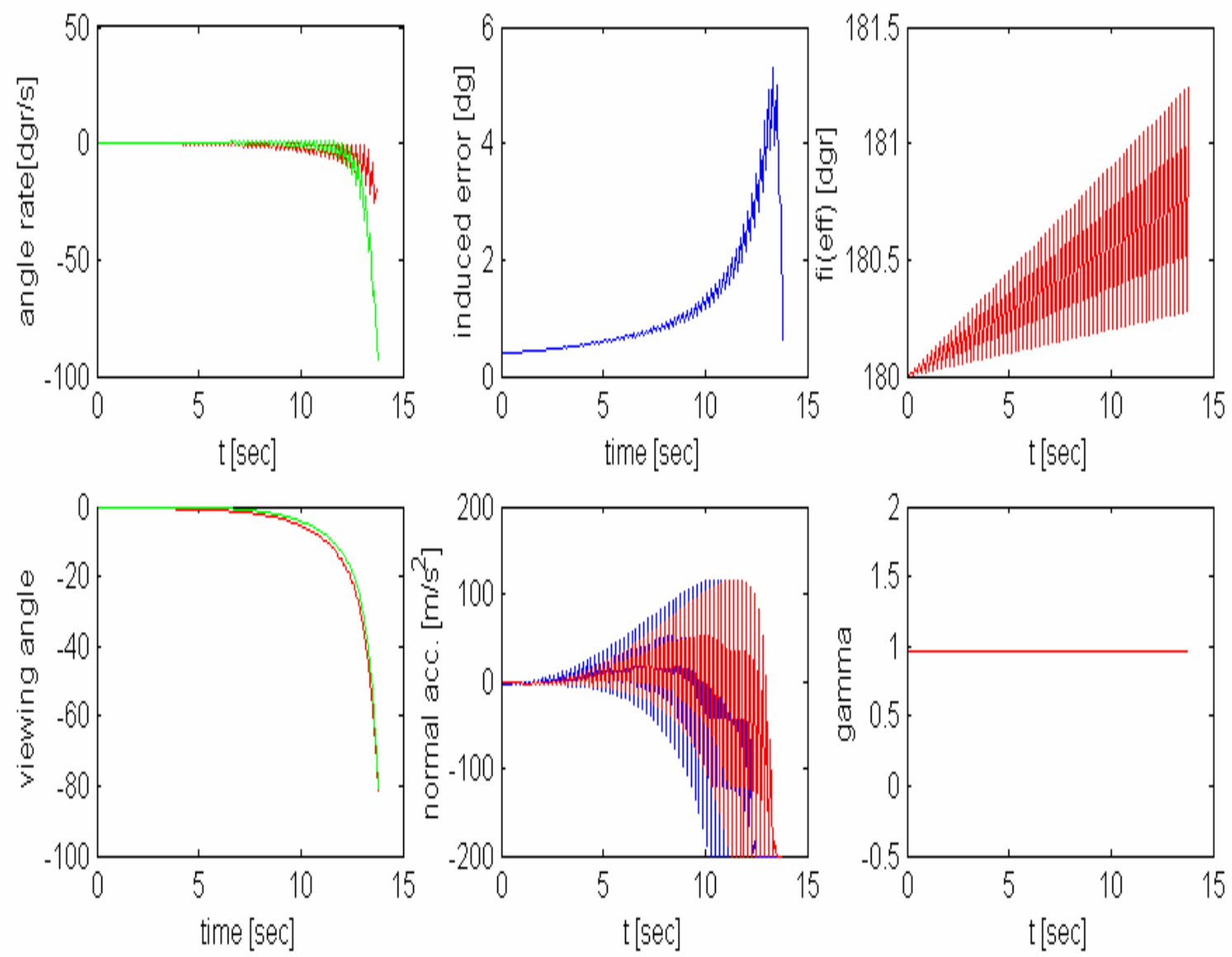

Fig. 5

The variation of Some Variables During the Run 